Spada et al.

\title{
Oxidative and non-oxidative active turnover of genomic methylcytosine in distinct pluripotent states
}

\author{
Fabio Spada ${ }^{1 *}$, Sarah Schiffers ${ }^{1}$, Angie Kirchner $^{1}$, Yingqian Zhang ${ }^{1,2}$, Gautier Arista $^{1}$, Olesea \\ Kosmatchev ${ }^{1}$, Eva Korytiakova ${ }^{1}$, René Rahimoff ${ }^{1 \S}$, Charlotte Ebert, and Thomas Carell ${ }^{1 *}$ \\ ${ }^{1}$ Department of Chemistry, Ludwig Maximilians University Munich and Center for Integrated \\ Protein Science Munich (CIPS ${ }^{\mathrm{M}}$ ) \\ ${ }^{2}$ College of Chemisty, State Key Laboratory of Elemento-Organic Chemistry and Department \\ of Chemical Biology, Nankai University, Tianjin, China \\ ${ }^{\S}$ Current addresses: S.S., National Cancer Institute, Center for Cancer Research, Bethesda, \\ MD, USA; A.K., Cancer Research UK Cambridge Institute, UK; R.R., Department of \\ Chemistry, University of California, Berkeley, CA, USA.
}

*Corresponding authors: Fabio Spada fabio.spada@cup.Imu.de; Thomas Carell: Thomas.Carell@cup.Imu.de

\begin{abstract}
Epigenetic plasticity underpins cell potency, but the extent to which active turnover of DNA methylation contributes to such plasticity is not known and the underlying pathways are poorly understood. Here we use metabolic labelling with stable isotopes and mass spectrometry to quantitatively address the global turnover of genomic methylcytidine (mdC), hydroxymethylcytidine ( $\mathrm{hmdC}$ ) and formylcytidine $(\mathrm{fdC})$ across mouse pluripotent cell states. High rates of $\mathrm{mdC} / \mathrm{hmdC}$ oxidation and $\mathrm{fdC}$ turnover characterize a formative-like pluripotent state. In primed pluripotent cells the global mdC turnover rate is about 3-6\% faster than can be explained by passive dilution through DNA synthesis. While this active component is largely dependent on Tet-mediated $\mathrm{mdC}$ oxidation, we unveiled an additional mdC oxidationindependent turnover process based on DNA repair. This process accelerates upon acquisition of primed pluripotency and returns to low levels in lineage committed cells. Thus, in pluripotent cells active $\mathrm{mdC}$ turnover involves both $\mathrm{mdC}$ oxidation-dependent and -independent processes.
\end{abstract}


Spada et al.

\section{Introduction}

Methylation at position 5 of deoxycytidine $(\mathrm{dC})$ is the most prominent post-replicative nucleobase modification in higher eukaryotic genomes and its functional relevance has been extensively investigated ${ }^{1,2}$. In mammals, genomic $\mathrm{dC}$ is methylated to 5-methyl-2'deoxycytidine $(\mathrm{mdC})$ by the DNA (cytosine-5)-methyltransferases DNMT1, DNMT3a and DNMT3b ${ }^{3}$, mostly, but not exclusively in the context of $\mathrm{CpG}$ dinucleotides ${ }^{4}$. These enzymes differentially contribute to establishing and maintaining genomic $\mathrm{mdC}$ patterns across cellular and organismal generations. In addition, genomic mdC can be further modified by iterative oxidation to 5-hydroxymethyl-, 5-formyl- and 5-carboxyl-dC (hmdC, fdC and cadC, respectively) through the action of Ten-Eleven Translocation (TET) di-oxygenases ${ }^{5-7}$. As several classes of DNA binding factors display differential affinity for their target sequences depending on the $\mathrm{dC}$ modification state ${ }^{2,1,8}$, genomic patterns of $\mathrm{dC}$ modification contribute to consolidation and propagation of gene expression states and chromatin structure, thus affecting fundamental processes such as cell fate determination and restriction, imprinting, $X$ chromosome inactivation and genome stability ${ }^{1,2}$.

In the last two decades methylation of genomic $\mathrm{dC}$ was shown to be rapidly removed in various biological contexts ${ }^{9}$. Several erasure mechanisms were proposed, including prevention of methylation maintenance, which results in passive dilution of the modified base through DNA replication, and active demethylation by enzymatic processes $^{9}$ (Fig. 1a). Among the latter oxidation of $\mathrm{mdC}$ to fdC or cadC by TET enzymes followed by base excision repair (BER) mediated by the DNA glycosylases TDG $^{9,10}$ and Neil1/2 11,12 is best characterized. Additional proposals invoke the involvement of other DNA repair pathways ${ }^{9}$ or direct $\mathrm{C}-\mathrm{C}$ bond cleavage ${ }^{13-16}$. A third line of proposal posits that genomic mdC and hmdC could be deaminated to thymidine (dT) and 5-hydroxymethyl-2'-deoxyuridine (hmdU), respectively, either by a cytosine deaminase of the AID/APOBEC family ${ }^{9}$ or by de novo DNA methyltransferases DNMT3a/b ${ }^{17}$. The resulting $T: G$ or $h m U: G$ mismatches would be then resolved by BER or non- canonical mismatch repair (ncMMR) ${ }^{18-21}$. Finally, it was suggested that unmodified $\mathrm{dC}$ could be deaminated by AID and that long patch BER or ncMMR could lead to indirect co-removal of adjacent ${ }^{\mathrm{m}} \mathrm{C}$ residues ${ }^{20-22}$. Currently, all these pathways are highly controversial, as there is no conclusive evidence for their functional relevance in vivo ${ }^{9}$. It is however conceivable that different pathways operate at different stages during development.

During early embryonic development, the epiblast transits through a spectrum of pluripotent states $^{23}$. This transition can be modelled by cultivating mouse pluripotent stem cells (mPSCs) under specific conditions and involves dynamic changes in expression and 
epigenetic landscapes, including a major global gain of $\mathrm{mdC}^{23}$. Conversely, reversion from partially and heterogeneously primed mPSC cultures to the naïve state involves downregulation of de novo methyltransferases and collapse of DNA methylation maintenance with consequent global and passive (Tet enzyme-independent) loss of genomic methylation ${ }^{24}$. In contrast, little is known about the extent and nature of the turnover of modified genomic cytosines during the forward, developmentally relevant transition from naïve to primed pluripotent states. Here, we used metabolic labelling with stable isotope standards for highly accurate mass spectrometry based quantification of the global genomic $\mathrm{mdC}$, hmdC and fdC turnover through a progression of pluripotent states. We show that upon acquisition of primed pluripotency roughly $3-6 \%$ of the methylome is actively turned over. Most of this active turnover is due to Tet-mediated mdC oxidation, while a minor part involves an unknown $\mathrm{mdC}$ oxidation-independent DNA repair process.

\section{Results}

\section{Global genomic maC oxidation kinetics decrease upon progression to primed pluripotency.}

We cultured mPSCs under specific conditions to model the progression of the embryonic epiblast from naïve to primed pluripotency and investigate the kinetics and turnover of $\mathrm{dC}$ modification during this transition. In order to monitor priming conditions, we first made use of a mPSC line expressing an Oct4-YFP reporter ${ }^{25}$. After adaptation to naïve conditions in the presence of Gsk3 inhibitor CHIR99021 (CHIR), Mek1/2 inhibitor PD0325901 (also known as $2 \mathrm{i}$ ) and LIF, the cells were plated at low density in serum-containing medium supplemented with CHIR and the Tankyrase inhibitor IWR1-endo (serum/CR) as previously described $^{26}$. Live cell microscopy over the course of 6 days without passaging showed that these cells rapidly formed monolayer colonies that retained a remarkably homogeneous nuclear YFP signal across the culture (Supplementary Fig. S1a), indicating that under these conditions mPSC cultures may not loose pluripotency. Similarly intense and homogeneous YFP signals were observed when the same reporter line was permanently primed to mouse Epiblast Stem Cells (mEpiSCs) in serum-free, chemically defined medium supplemented with CHIR, IWR1, FGF-2 and Activin A (CDM/CRFA; Supplementary Fig. S1a). Expression analysis of pluripotency factors in serum/CR cultures and CDM/CRFA EpiSCs revealed similarly reduced and increased transcript levels of naïve and primed pluripotency factors, respectively (Supplementary Fig. S1b), showing that these conditions support similarly primed states. Thus, even without prolonged passaging or in chemically defined conditions, $\mathrm{CHIR/IWR} 1$ treatment supports primed pluripotency. We then used ultra-high pressure liquid 
chromatography coupled with triple quadrupole mass spectrometry (UHPLC-MS $\left.{ }^{2}\right)^{27}$ to analyse global levels of genomic $\mathrm{mdC}$ and $\mathrm{hmdC}$ upon priming of mPSCs under the same conditions over a five day time course. As previously reported ${ }^{28}$, the transition from the naïve (2i/LIF) to a homogeneously primed pluripotent state was marked by a large increase in global levels of genomic mdC, while hmdC abundance dropped sharply, both modifications reaching plateau levels by day 4 (Supplementary Fig. S2a). Global levels of genomic mdC and $\mathrm{hmdC}$ similar to those in serum/CR cultures were detected in Oct4-YFP mEpiSCs under serum-free conditions, excluding potential confounding effects of undefined serum composition. Note that under both serum-containing and serum-free conditions we avoided supplementation with ascorbic acid, which was shown to greatly increase the levels of oxidized $\mathrm{mdC}$ derivatives ${ }^{29}$. The decrease in $\mathrm{hmdC}$ abundance upon priming is consistent with a recent proteomic analysis showing that the levels of chromatin bound Tet1 and 2 proteins are markedly reduced in mEpiSCs compared to naïve mPSCs, while Tet3 protein is undetectable in both states ${ }^{30}$.

Notably, under 2i/LIF conditions, mPSCs exhibit low levels of genomic cytosine modification $^{23}$, while replacement of the Mek inhibitor with the Src inhibitor CGP77675 (conditions referred to as alternative $2 \mathrm{i}$ or a2i) results in relatively high genomic $\mathrm{mdC}$ levels ${ }^{31,32}$. Nevertheless, gene expression patterns remain similar and developmental potential unaltered relative to those of naïve $\mathrm{mPSCs}^{31,32}$. In particular, our expression analysis showed that the pattern of pluripotency and early lineage specification factors in mPSCs under a2i/LIF conditions was intermediate to that of naïve and primed states, with still high levels of Tfcp2l1 and KIf4 transcripts relative to 2i/LIF (naïve) conditions, but intermediate expression of Rex1, Stella and Prdm14 as well as low levels of Fgf5, Oct6, Otx2 and Dnmt3b relative to primed conditions (Fig. S1b). This pattern is highly similar to that previously shown for formative pluripotency, an intermediate state along the trajectory from naïve to primed pluripotency, during which competence for multi-lineage commitment is acquired $^{33}$. In addition, high depth bisulfite amplicon sequencing revealed increased de novo methylation at secondary imprints in the transition from a2i/LIF to serum/CR conditions (Supplementary Fig. S2b), which is similar to the progression previously shown from pre- to early post-implantation stages in mouse embryos ${ }^{34}$. Altogether, our expression and genomic methylation analyses support that cultures transited from a2i/LIF to serum/CR conditions recapitulate fundamental molecular processes of the progression to a genuine primed pluripotent state. Importantly, prolonged culture under 2i/LIF conditions leads to loss of imprinted methylation and genetic instability, both are persevered in a2i/LIF long term cultures $^{31,32}$. In addition, a2i/LIF and serum/CR conditions allow comparison of genomic cytosine modification dynamics between functionally distinct pluripotent states with 
substantial and comparable steady state levels of genomic mdC. Thus, we adopted a2i/LIF conditions both for routine mPSC maintenance and as starting state for further time course analyses of mPSC priming.

We then set out to monitor global cytosine modification kinetics during acquisition of primed pluripotency by metabolic labelling of the C5 substituent. To this aim we supplemented L-Methionine (Met)-free medium with isotopically labelled Met $(m+4 M e t)$, whereby the methyl group provides a mass shift of four units $\left(\left[\right.\right.$ methyl- $\left.{ }^{13} \mathrm{C}, \mathrm{d}_{3}\right]$, hereafter referred to as $\left.\mathrm{m}+4\right)$. As for naturally occurring Met, $m+4$ Met is incorporated into S-adenosylmethionine (SAM) and $m+4$ is transferred thereof onto genomic $d C$ by Dnmts, generating $m+4 d C$. The latter can be sequentially oxidised by Tet proteins to $h m+3 d C, f+2 d C$ and ca+1dC (Fig. 1a) $)^{27}$.

In order to attain accurate absolute quantification of both naturally occurring (mdC, hmdC, $f d C)$ and heavy $(m+4 d C, h m+3 d C, f+2 d C)$ isotopologues, we synthesised an even heavier series of corresponding isotopologues $\left(\left[\mathrm{d}_{4},{ }^{15} \mathrm{~N}_{2}\right]-\mathrm{mdC},\left[{ }^{13} \mathrm{C}_{5},{ }^{15} \mathrm{~N}_{2}\right]-\mathrm{hmdC}\right.$ and $\left[{ }^{13} \mathrm{C}_{5},{ }^{15} \mathrm{~N}_{2}\right]-\mathrm{fdC}$; Fig. 1b) and used them as internal standards for quantification by UHPLC-MS ${ }^{2}$. In this work cadC and ca+1dC were not considered as isotopologues differing by only one mass unit cannot be reliably discriminated using UHPLC-MS ${ }^{2}$. We first compared the modification kinetics in cultures maintained constantly under a2i/LIF conditions to cultures undergoing priming in CHIR/IWR1 medium. In both cases we performed time course analyses over 2 days of labelling in the presence of $m+4 M e t$, which in the case of priming cultures was from day 3 to day 5 after transfer to serum/CR medium (Fig.s 1c,d, Supplementary Fig. S3). Relative to a2i/LIF conditions, in priming cultures the rate of $\mathrm{m}+4 \mathrm{dC}$ oxidation to $\mathrm{hm}+3 \mathrm{dC}$ was clearly slower. Accumulation of the further oxidized state $f+2 d C$ became undetectable under these conditions. Thus, the transition to primed pluripotency determines a major decrease in global oxidation of genomic $\mathrm{mdC}$, which is consistent with the drastically reduced steady state levels of genomic hmdC (Supplementary Fig. S2a) and with a recent report of lower levels of chromatin bound Tet proteins in primed cultures ${ }^{30}$

\section{An active and largely Tet enzyme-dependent component of genomic mdC turnover in primed pluripotent cells}

To monitor the turnover of modified genomic cytosines we reversed the labelling schema described above. We first pulsed labelled cultures with $\mathrm{m}+4 \mathrm{Met}$, then chased the label for two days by transferring the cultures to medium with naturally occurring Met and collected samples every $12 \mathrm{~h}$ during the chase (Fig. 2a). We again compared cultures maintained constantly under serum/a2i/LIF conditions to cultures undergoing priming in serum/CR medium. In both cases the cultures were pulsed for five days with $m+4$ Met before chasing. 
In the case of the primed cultures the first two pulse days were in a2i/LIF medium, while the last three were in serum/CR medium, thus corresponding to the first three days of priming. To generate a reference for passive dilution of a genomic nucleoside through DNA synthesis, we co-pulsed with an isotopically labelled 2'-deoxythymidine having a mass shift of 12 units $\left(\left[{ }^{15} \mathrm{~N}_{2},{ }^{13} \mathrm{C}_{10}\right]\right.$-dT; hereafter referred to as $\mathrm{dT}+12$; Fig. 2a). Due to its highly efficient incorporation, $\mathrm{dT}+12$ was co-pulsed only during the last day of the m+4Met pulse. Thereafter, $\mathrm{dT}+12$ was chased for two days in parallel to $\mathrm{m}+4$ Met. Remarkably, while in most cases the levels of modified cytosines readily declined with the onset of the chase, under a2i/LIF conditions $h m+3 d C$ and $f+2 d C$ accumulated for the first 12 and 24 hours, respectively (Fig. 2b). This shows again that the rate of Tet-mediated oxidation is quite high in mPSCs under a2i/LIF conditions and declines sharply upon priming, as already seen with the time course analysis during $\mathrm{m}+4 \mathrm{Met}$ labelling (Fig. 1b,c). Notably, under a2i/LIF conditions the decline of $\mathrm{f}+2 \mathrm{dC}$ after $36 \mathrm{~h}$ became quite steep, pointing to high turnover of $\mathrm{fdC}$. Although under priming conditions oxidation of $\mathrm{mdC}$ to $\mathrm{hmdC}$ and further to fdC was hardly detectable in labelling time course experiments (Fig. 1), the fact that in the pulse chase experiments the decline of labelled $\mathrm{hmdC}$ and $\mathrm{fdC}$ was slower than that of $\mathrm{dT}+12$ clearly shows that some $\mathrm{mdC}$ and $\mathrm{hmdC}$ oxidation still takes place (Fig $2 \mathrm{~b}$, central panel). Importantly, upon priming, the turnover of $m+4 d C$ is faster than the dilution rate of $d T+12$ (Fig. 2b, inset), revealing the presence of a component of the $m+4 d C$ turnover that cannot be explained by passive dilution through DNA synthesis.

To assess the contribution of Tet-dependent oxidation to this component we performed the same pulse/chase experiment under priming condition (Fig. 2a, upper part) with E14tg2a mPSCs lacking all three Tet proteins (Tet triple knockout, alias Tet TKO) ${ }^{35}$ as well as parental, Tet 1-3 proficient E14tg2a cells (Fig. 3; note that E14tg2a cells are Hprt null ${ }^{36}$ ). In the parental cell line (Tet1-3 proficient), turnover of genomic $\mathrm{m}+4 \mathrm{dC}$ was again clearly faster than passive dilution of $d T+12$. After averaging the data from three such pulse chase experiments with wt $\mathrm{mPSC}$ lines, a paired two-tailed T-test showed high significance $(\mathrm{P}=$ $0.0053)$ for the deviation between $m+4 d C$ and $d T+12$ decay rates. This accounts for an active component of global $\mathrm{mdC}$ turnover involving $3-6 \%$ of the methylome, depending on the time point (Fig. 2c). In contrast, in the genome of Tet TKO cells the decline rate of $m+4 d C$ was essentially indistinguishable from that of $d T+12$ (Fig. 3). This shows that the active component of the $\mathrm{mdC}$ turnover is indeed largely dependent on the presence of Tet enzymes, and thus mostly due to mdC oxidation.

\section{Additional mdC turnover through a DNA repair process}


Next to oxidative mdC turnover, we started to investigate a potential deamination based pathway. Deamination of $\mathrm{mdC}$ and $\mathrm{hmdC}$ to $\mathrm{dT}(\mathrm{mdU})$ and hmdU, respectively, has been proposed as a potential alternative first step for active erasure of genomic $\mathrm{C}$ methylation. Upon metabolic labelling with $m+4$ Met, deamination of $m+4 d C$ would generate $m+4 d U$, that is dT bearing the $\mathrm{m}+4$ methyl group (Fig. 1a). In order to investigate this process quantitatively we synthesised the isotopologue $\mathrm{dT}-\left[{ }^{13} \mathrm{C}_{5},{ }^{15} \mathrm{~N}_{2}\right]$ (Fig. 1b) and used it as a standard for UHPLC-MS ${ }^{2}$ analysis. As the source of methyl group for de novo dT biosynthesis is serine through 5,10-methylenetetrahydrofolate and not methionine through SAM, it is expected that, upon supplementation of the medium with $m+4 M e t, m+4 d C$ is the only source of $\mathrm{m}+4 \mathrm{dU}$. To verify this expectation, we made use of mPSCs lacking all catalytically active Dnmts (Dnmt TKO), and therefore devoid of genomic $\mathrm{mdC}^{37}$. After priming in the presence of $\mathrm{m}+4 \mathrm{Met}, \mathrm{m}+4 \mathrm{dU}$ was clearly detected in the genome of parental wt $\mathrm{J} 1 \mathrm{mPSCs}$, but not in Dnmt TKO cultures. Thus, generation of $\mathrm{m}+4 \mathrm{dU}$ strictly requires the presence of $\mathrm{m}+4 \mathrm{dC}$, showing that $\mathrm{m}+4 \mathrm{dU}$ originates exclusively through deamination of $m+4 d C$ (Supplementary Fig. S4).

Direct deamination of genomic $\mathrm{mdC}$ would imply the formation of $\mathrm{T}: \mathrm{G}$ mismatches, which, if not efficiently repaired, would be highly mutagenic. It would then be reasonable to expect that a mechanism for highly efficient coupling of $\mathrm{mdC}$ deamination with BER of the ensuing $\mathrm{T}: \mathrm{G}$ mismatches would have co-evolved ${ }^{19}$ so as to minimize the steady state levels of such mismatches. In contrast to this idea, we surprisingly detected substantial accumulation of $m+4 d U$ in genomic DNA, whenever cultures were provided with m+4Met (gDNA; Fig. 1-5, Supplementary Fig. S3-4, S7). To explain this observation we considered an alternative scenario whereby genomic strands containing $\mathrm{m}+4 \mathrm{dC}$ may first be subject to a DNA repair process leading to the release of soluble $m+4 d C / m+4 d C M P$, either by direct exonuclease action, as upon MMR and end resection of double strand breaks, or through digestion of excised strands (e.g., in nucleotide excision repair). Deamination of this material by Cytidine and Deoxycytidylate deaminases (Cda and $\mathrm{Dctd})^{38}$, would generate soluble $m+4 d U / m+4 d U M P$, which could be phosphorylated and re-incorporated into the genome through DNA synthesis (Fig. 4a). To test if the high levels of $m+4 d U$ detected in the genome are generated along this pathway, we supplemented the medium with $m+4 \mathrm{Met}$ and increasing concentrations of unlabelled $\mathrm{dT}$. If DNA repair generates soluble $m+4 d U / m+4 d U M P$, incorporation of the latter into gDNA will be outcompeted by unlabelled dT. In addition, a dT excess will lead to high concentrations of dTMP and dTTP, which are both known to feedback negatively on Dctd through product inhibition and allosteric regulation, respectively ${ }^{39}$. The accumulation of genomic $\mathrm{m}+4 \mathrm{dU}$ in priming mPSC cultures was indeed progressively reduced with supplementation of increasing concentrations of 
unlabelled dT (Fig. 4b). This result supports the idea that genomic $m+4 d U$ originates in large part from a pool of soluble precursor.

To further probe whether deamination of $m+4 d C$ takes place within gDNA or at the level of soluble nucleoside/nucleotide pools, we next used CRISPR/Cas9-mediated gene editing to generate mPSC with double deficiency for Cda and Dctd (CD-DKO; Supplementary Fig. $5 a, b)$. Genomic sequencing showed that three of the isolated clonal lines bear compound biallelic Cda and Dctd mutations incompatible with the expression of functional enzymes (Supplementary Fig S5c-e). All three CD-DKO clones grew at slower rates than parental wt cells, a phenotype which was at least partially relieved by supplementation of the medium with dT (Supplementary Fig. S5f). This indicates that deamination of $(d) C /(d) C M P$ by Cda and/or Dctd provides substantial amounts of (d)U/(d)UMP for biosynthesis of (d)T/(d)TMP by Thymidine synthase to support DNA replication and is fully consistent with previously reported dT auxotrophy of Dctd deficient somatic cell lines ${ }^{40}$. As two of the CD-DKO clones grew particularly poorly under serum/CR priming conditions, we first used serum/LIF medium for metabolic labelling with $\mathrm{m}+4$ Met under priming conditions. Importantly, while the genomic content of $\mathrm{mdC} / \mathrm{m}+4 \mathrm{dC}$ was the same as in parental wt $\mathrm{J} 1$ cells, only background levels of $\mathrm{m}+4 \mathrm{dU}$ could be measured in all three CD-DKO clones (Fig 4c). The same result was obtained with CD-DKO clone 40 upon priming in serum/CHIR/IWR1 medium (Fig. 4d). These results clearly show that the genomic $m+4 d U$ measurable in wt cells with our UHPLC-MS ${ }^{2}$ method originates from a process involving direct repair of gDNA containing $m+4 \mathrm{dC}$, with consequent release of soluble $m+4 d C / m+4 d C M P$, followed by deamination to $m+4 d U / m+4 d U M P$ and (re)-incorporation of the latter into the genome through phosphorylation to the triphosphate state and DNA synthesis. Although our data point to exclusive deamination in the soluble pool, we wanted to further investigate potential deamination events of $\mathrm{mdC}$ to $\mathrm{T}$ directly in the genome, particularly in light of previous reports in this direction ${ }^{17,19}$. In this respect it is important to consider the limitations of our quantitative UHPLC-MS ${ }^{2}$ method. In UHPLC the overwhelming amount of natural dT present in the genome co-elutes with $\mathrm{m}+4 \mathrm{dU}$ and potentially outcompetes it during ionization for the capture of charge. The effect is a strong ion suppression, resulting in a reduction of the $m+4 \mathrm{dU}$ signal and, consequently, a high limit of detection (LOD). To put this effect into perspective we calculated that our LOD for $\mathrm{m}+4 \mathrm{dU}$ is about 100 times higher than that for fdC (around $1 \times 10^{-5}$ versus $1 \times 10^{-7}$ per nucleotide), which is a relatively stable $\mathrm{dC}$ modification considered to have an epigenetically relevant role.

However, deamination of very few $m+4 d C$ residues directly within the genome could trigger long patch BER and/or $M M R^{20,21}$, leading to the release of much larger amounts of $m+4 d C$ in the soluble pool and, subsequently, deamination to $\mathrm{m}+4 \mathrm{dU}$ and re-incorporation into the 
genome, explaining the large amounts of genomic $\mathrm{m}+4 \mathrm{dU}$ observed. In CD-DKO cells direct deamination of genomic $\mathrm{m}+4 \mathrm{dC}$ would be expected to be the only potential source of $\mathrm{m}+4 \mathrm{dU}$ and, as the latter would then be mismatched to $G$, its steady state levels would be expected to be very low due to efficient repair. In order to probe genomic deamination with higher sensitivity the ion suppression effect had to be substantially reduced. To this end we performed a double labelling experiment with $\mathrm{m}+4 \mathrm{Met}$ and a $\mathrm{dC}$ nucleoside where 9 hydrogen atoms are replaced with deuterium ( $d C\left[D_{9}\right]$; Supplementary Fig. S5a). After incorporation into the genome, $d C\left[D_{9}\right]$ will be methylated to $m+4 d C\left[D_{8}\right]$, which deaminates to $m+4 d U\left[D_{8}\right]$ (a dT with eleven $D$ atoms and $a+12$ mass shift; Supplementary Fig S6a). This massive H-to-D exchange was chosen as it is known to determine a shift to shorter retention time in UHPLC, allowing $m+4 d U\left[D_{8}\right]$ to escape co-elution with $d T$. Indeed, co-injection tests showed a slightly shorter retention time for $\mathrm{dT}\left[\mathrm{D}_{8}\right]$ relative to $\mathrm{dT}$ (Supplementary Fig. S6b). Notably, $m+4 d U\left[D_{8}\right]$ is expected to have an even shorter retention time that would allow it to at least partly escape ion suppression from natural $d T$. After double labelling with $m+4 \mathrm{Met}$ and $d C\left[D_{9}\right]$ we were able to detect $m+4 d U\left[D_{8}\right]$ confidently only in wt mPSCs (Supplementary Fig. S6c). However, we sporadically detected small $m+4 d U\left[D_{8}\right]$ peaks in technical replicates of CD-DKO samples. After integration of these sporadic signals, the corresponding values were invariably below LOD, defined as three times the average level of blank samples. Although we cannot exclude that these signals are caused by background processes, we nevertheless performed a calculation taking into account the limits of detection and quantification that were gained by deuterium labelling. This calculation allows us to estimate the maximum number of potential genomic deamination events between 500 and 5000 $m+4 d U\left[D_{8}\right]$ molecules per cell in the course of our labelling experiment. According to an estimated rate constant for spontaneous hydrolytic deamination of $\mathrm{mdC}$ in solution ${ }^{41}$ only around 20 such events would be expected to take place. Therefore, although a reliable and reproducible signal for $m+4 d U\left[D_{8}\right]$ could not be detected in CD-DKO cells, we cannot exclude the occurrence of genomic deamination above the rates of spontaneous hydrolysis. However, in the best case this process cannot exceed a level of $1 \times 10^{-6}-1 \times 10^{-7}$ events per nucleotide.

\section{Tet enzymes do not trigger the DNA repair process responsible for turnover of genomic mdC into dT}

Conversion of $\mathrm{m}+4 \mathrm{dC}$ into $\mathrm{m}+4 \mathrm{dU}$ obviously does not require oxidized intermediates. However, it was recently suggested that in the zygote Tet3 generates undefined DNA lesions, triggering repair-mediated erasure of genomic methylation from the paternal genome $^{42}$. Also, hmdC may mark DNA damage sites and promote their repair ${ }^{43}$ or even 
target DNA repair by selectively recruiting endonucleases like Srap $1^{44}$ and Endonuclease $\mathrm{G}^{45}$. We therefore considered whether Tet proteins contribute to the turnover of genomic mdC into dT by generating hmdC or low levels of DNA lesions such as hmdU ${ }^{27}$, which triggers BER initiated by the DNA glycosylase Smug1. To this aim we primed Tet TKO and parental E14tg2a mESCs in the presence of $m+4$ Met. Surprisingly, higher levels of genomic $\mathrm{m}+4 \mathrm{dU}$ were detected in the absence of Tet enzymes (Fig. S7). Similarly, analysis of the pulse chase experiment with the same pair of cell lines also revealed a tendency for higher accumulation of $\mathrm{m}+4 \mathrm{dU}$ in Tet TKO mPSCs at later chase time points (Fig. 3, lower panels). Therefore, in pluripotent cells Tet proteins do not seem to trigger DNA repair events that contribute to turnover of $\mathrm{mdC}$ into $\mathrm{dT}$.

\section{Oxidation-independent repair of mdC-containing gDNA is developmentally regulated}

To investigate whether oxidation-independent mdC turnover is regulated during the transition to primed pluripotency, we first compared the genomic abundance of $m+4 d U$ in the time course and pulse-chase experiments under naïve and priming conditions described above (Fig. 1-3, Supplementary Fig. S3). In time course experiments, progressive accumulation $\mathrm{m}+4 \mathrm{dU}$ was much faster under priming conditions, reaching approximately five times higher levels than under naïve conditions (Fig. 1, Supplementary Fig. S3). Also, m+4dU was the only $\mathrm{m}+4 \mathrm{dC}$ derivative that accumulated during label chase under priming conditions, though to different extents in the two cell lines tested (Fig. 2b,3). Thus, in contrast with the observed decline of mdC oxidation (Fig. 1,2, Supplementary Fig. S3), these results reveal increased rates of oxidation-independent repair of mdC-containing gDNA upon acquisition of primed pluripotency. This anti-correlation suggests that oxidation-independent $\mathrm{mdC}$ turnover through DNA repair may serve at least in part as a supplementary pathway for the erasure of dC methylation. However, as in primed Tet TKO mPSCs there was no apparent difference between global mdC turnover and passive dilution through DNA synthesis (Fig. 3: decay of $m+4 d C$ and $d T+12$, respectively), this process cannot involve more than $1 \%$ of the methylome.

At last, we compared oxidation-independent $\mathrm{mdC}$ turnover through DNA repair in pluripotent states (intermediate serum/a2i/LF as well as primed serum/CR and EpiSCs under $\mathrm{CDM} / \mathrm{CHIR/IWR1/FGF-2/Activin} \mathrm{A} \mathrm{conditions)} \mathrm{with} \mathrm{various} \mathrm{lineage} \mathrm{committed} \mathrm{cell} \mathrm{types,}$ including primary and immortalized embryonic fibroblasts, neural stem cells (NSCs) ${ }^{46}$ and HeLa cells. Although primed pluripotent cells were labelled with $\mathrm{m}+4 \mathrm{Met}$ for only two to three days, $\mathrm{m}+4 \mathrm{dU}$ reached higher levels in their genome than in those of any of the somatic cell types cultured for substantially longer periods, ranging from 6 to 12 days (Fig. 5a). This 
clearly shows that in lineage committed cells oxidation-independent mdC turnover through DNA repair is substantially lower than in primed pluripotent cells. In addition, the rate of oxidation-independent $\mathrm{mdC}$ turnover is clearly not proportional to the global genomic abundance of $\mathrm{mdC}$ either between pluripotent states or among pluripotent and lineage committed cells. In particular, among the latter oxidation-independent mdC turnover is clearly more active in NSCs than in fibroblasts, despite the mdC genomic content being at least $20 \%$ lower in NSCs. These observations indicate that oxidation-independent $\mathrm{mdC}$ turnover is under developmental and cell lineage-dependent control.

\section{Discussion}

Recent methylome analysis of matched clonal and polyclonal cell populations ${ }^{47}$ and studies on the contribution of Dnmt3 proteins to DNA methylation maintenance ${ }^{48,49}$ suggested that in heterogeneously primed mPSCs cultures (serum/LIF) genomic mdC undergoes higher dynamic turnover as compared to differentiated somatic cells. However, the extent and especially the nature of this turnover have not been addressed. Previously, Bachmann et al. reported pulse-chase experiments with $m+4$ Met to estimate the global turnover of genomic dC modifications in mPSCs cultured under naïve conditions (2i/LIF), using a very short pulse time ${ }^{50,51}$. However, the naïve pluripotent state is characterised by very low global levels of genomic dC modifications, which increase only upon exit from the naïve state and acquisition of primed pluripotency ${ }^{1,23}$, a crucial transition to lay the ground for commitment to somatic lineages.

Here, we report the first analysis of on- and off-rate kinetics for genomic $\mathrm{dC}$ modifications upon transition to primed pluripotency. We show that in mPSCs under a2i/LIF conditions, which display expression and genomic methylation patterns that are intermediate between naïve and primed pluripotent states and resemble those reported for the formative state ${ }^{33}$, the rates of $\mathrm{mdC}$ oxidation to $\mathrm{hmdC}$ and further to $\mathrm{fdC}$ are quite sustained and the removal/conversion of $\mathrm{fdC}$ is very rapid. This points to a very dynamic equilibrium for $\mathrm{fdC}$ under these conditions. It is tempting to speculate that these high levels of oxidative turnover may underlie the frequent periodical oscillations in global mdC levels recently identified through single cell analysis in partially (and heterogeneously) primed serum/LIF cultures ${ }^{52}$. In contrast to the high levels of $\mathrm{mdC}$ oxidation in a2i/LIF conditions, upon transition to a more advanced, primed pluripotency state the global oxidation rates of $\mathrm{mdC}$ and $\mathrm{hmdC}$ as well as the removal of hmdC and fdC decline drastically. Despite this the global turnover rate of genomic $\mathrm{mdC}$ is $3-6 \%$ faster than can be accounted for by passive dilution through DNA synthesis (DNA replication and repair; Fig. 5b) and this difference is largely due to Tetmediated $\mathrm{mC}$ oxidation. Evidence for passive erasure of $\mathrm{dC}$ methylation based on impairment of its maintenance has been provided for primordial germ cells, preimplantation 
embryonic development and the reversion from heterogeneous partially primed/formative states present in serum/LIF cultures to the naïve pluripotent state ${ }^{1,24}$. We suggest that against the backdrop of global gain and maintenance of $\mathrm{dC}$ methylation occurring in the forward developmental transition from naïve to primed pluripotency an active demethylation mechanism may be more attainable than preventing maintenance of $\mathrm{dC}$ methylation at selected sites, especially in the case of loci with a highly accessible chromatin conformation like actively transcribed genes.

Furthermore, by tracing the origin of genomic $m+4 d U$ to deamination of soluble $m+4 d C$, we show that a small part of genomic mdC is turned over by a DNA repair process that involves its direct excision from the genome (Fig. 5b). While we show that TET proteins are clearly not involved in triggering or targeting this non-oxidative $\mathrm{mdC}$ turnover, our data neither support nor rule out that low levels of direct enzymatic deamination of genomic mdC may trigger the DNA repair process underlying this turnover pathway. Although the nature of this DNA repair process and the events triggering it remain to be determined, its rate is not proportional to global genomic mdC levels, either between pluripotent states or between lineage committed cells, indicating that the underlying DNA repair process is developmentally regulated. In this regard, it should be noted that in PSCs, including primed human PSCs, the expression of DNA repair factors and the proficiency of various DNA repair pathways were shown to be higher than in somatic cells ${ }^{53-55}$. Interestingly, the extent of oxidation-independent turnover of $\mathrm{mdC}$ through DNA repair is not proportional to global genomic mdC levels, either between pluripotent states or lineage committed cells, indicating that the underlying DNA repair process does not take place randomly throughout the methylome. In addition, the levels of oxidation-independent mdC turnover show an inverse correlation with $\mathrm{mdC}$ oxidation rates across pluripotent states and seem to be slightly more sustained in Tet deficient cells, pointing to a possible function as alternative or supplementary mechanism dedicated to active erasure of cytosine methylation. Whether this is indeed the case requires further investigation.

\section{Acknowledgements}

We are very thankful to the following colleagues: Markus Möser (Max Planck Institute for Biochemistry, Martinsried, Germany) for K3 mPSCs (Kindlin3+/+); Hitoshi Niwa and Masaki Okano (both at Kumamoto University, Japan) for Oct4-YFP-Puro mPSCs and Dnmt TKO J1, respectively; Yi Zhang (Boston Children's Hospital, Boston, MA) for parental and Tet TKO E14tg2a. Anti-DCTD antibody and purified recombinant DCTD protein were generous gifts from Frank Maley (New York State University, NY), Sebastian Bultmann and Christopher 
Mulholland for guidance on high depth bisulfite amplicon sequencing (both at Ludwig Maximilian University, Munich).

Funding was provided by the Deutsche Forschungsgemeinschaft via the programs SFB1309 (TP: A4), SFB1361 (TP: 2), GRK 2338 and SPP-1784. This project has received funding from the European Research Council (ERC) under the European Union's Horizon 2020 research and innovation programme (grant agreement $\left.n^{\circ} E P i R 741912\right)$. $Y Z$ is supported by the China Scholarship Council (CSC Nr. 201806200069).

\section{Author Contributions}

FS and TC conceived the study and designed experiments. FS, SS, AK, YZ, GA, OK and JS performed experiments and analyzed data. FS, TC and SS interpreted data. RR, CE and EK synthesized isotopically labelled nucleosides used as standards for LC-MS/MS. FS, TC and SS wrote the manuscript.

\section{Competing interests}

The authors declare no competing interests.

\section{Data availability and code availability statements}

The raw data that support the findings of this study are available from the corresponding authors upon reasonable request. No own code was used. 
bioRxiv preprint doi: https://doi.org/10.1101/846584; this version posted March 9, 2020. The copyright holder for this preprint (which was not certified by peer review) is the author/funder, who has granted bioRxiv a license to display the preprint in perpetuity. It is made available under aCC-BY 4.0 International license.

Spada et al.

\section{References}

1. Greenberg, M. V. C. \& Bourc'his, D. The diverse roles of DNA methylation in mammalian development and disease. Nat Rev Mol Cell Biol 1-18 (2019) doi:10.1038/s41580-019-0159-6.

2. Luo, C., Hajkova, P. \& Ecker, J. R. Dynamic DNA methylation: In the right place at the right time. Science 361, 1336-1340 (2018).

3. Jeltsch, A. \& Jurkowska, R. Z. Allosteric control of mammalian DNA methyltransferases - a new regulatory paradigm. Nucl. Acids Res. 44, 8556-8575 (2016).

4. He, Y. \& Ecker, J. R. Non-CG Methylation in the Human Genome. Annual Review of Genomics and Human Genetics 16, 55-77 (2015).

5. Tahiliani, M. et al. Conversion of 5-Methylcytosine to 5-Hydroxymethylcytosine in Mammalian DNA by MLL Partner TET1. Science 324, 930-935 (2009).

6. Ito, S. et al. Tet Proteins Can Convert 5-Methylcytosine to 5-Formylcytosine and 5-Carboxylcytosine. Science 333, 1300-1303 (2011).

7. He, Y.-F. et al. Tet-Mediated Formation of 5-Carboxylcytosine and Its Excision by TDG in Mammalian DNA. Science 333, 1303-1307 (2011).

8. Spruijt, C. G. et al. Dynamic Readers for 5-(Hydroxy)Methylcytosine and Its Oxidized Derivatives. Cell 152, 1146-1159 (2013).

9. Schuermann, D., Weber, A. R. \& Schär, P. Active DNA demethylation by DNA repair: Facts and uncertainties. DNA Repair 44, 92-102 (2016).

10. Wu, X. \& Zhang, Y. TET-mediated active DNA demethylation: mechanism, function and beyond. Nat Rev Genet 18, 517-534 (2017).

11. Slyvka, A., Mierzejewska, K. \& Bochtler, M. Nei-like 1 (NEIL1) excises 5-carboxylcytosine directly and stimulates TDG-mediated 5-formyl and 5-carboxylcytosine excision. Scientific Reports 7, 9001 (2017).

12. Schomacher, L. et al. Neil DNA glycosylases promote substrate turnover by Tdg during DNA demethylation. Nature Structural \& Molecular Biology 23, 116-124 (2016).

13. Chen, C.-C., Wang, K.-Y. \& Shen, C.-K. J. The Mammalian de novo DNA Methyltransferases Dnmt3a and Dnmt3b Are Also DNA 5-Hydroxymethyl Cytosine Dehydroxymethylases. J. Biol. Chem. 287, 33116-33121 (2012).

14. Liutkevičiūtè, Z., Lukinavičius, G., Masevičius, V., Daujotytè, D. \& Klimašauskas, S. Cytosine-5methyltransferases add aldehydes to DNA. Nature Chemical Biology 5, 400-402 (2009).

15. Iwan, K. et al. 5-Formylcytosine to cytosine conversion by $\mathrm{C}-\mathrm{C}$ bond cleavage in vivo. Nature Chemical Biology 14, 72-78 (2017).

16. Schön, A. et al. Analysis of an active deformylation mechanism of 5-formyl-deoxycytidine (fdC) in stem cells. Angewandte Chemie International Edition AOP (2020) doi:10.1002/anie.202000414.

17. Metivier, R. et al. Cyclical DNA methylation of a transcriptionally active promoter. Nature 452, 45-50 (2008).

18. Cortellino, S. et al. Thymine DNA Glycosylase Is Essential for Active DNA Demethylation by Linked Deamination-Base Excision Repair. Cell 146, 67-79 (2011).

19. Rai, K. et al. DNA Demethylation in Zebrafish Involves the Coupling of a Deaminase, a Glycosylase, and Gadd45. Cell 135, 1201-1212 (2008).

20. Grin, I. \& Ishchenko, A. A. An interplay of the base excision repair and mismatch repair pathways in active DNA demethylation. Nucl. Acids Res. 44, 3713-3727 (2016).

21. Franchini, D.-M. et al. Processive DNA Demethylation via DNA Deaminase-Induced Lesion Resolution. PLoS ONE 9, e97754 (2014).

22. Santos, F. et al. Active demethylation in mouse zygotes involves cytosine deamination and base excision repair. Epigenetics \& Chromatin 6, 39 (2013).

23. Atlasi, Y. \& Stunnenberg, H. G. The interplay of epigenetic marks during stem cell differentiation and development. Nature Reviews Genetics 18, 643-658 (2017).

24. von Meyenn, F. et al. Impairment of DNA Methylation Maintenance Is the Main Cause of Global Demethylation in Naive Embryonic Stem Cells. Molecular Cell 62, 848-861 (2016).

25. Toyooka, Y., Shimosato, D., Murakami, K., Takahashi, K. \& Niwa, H. Identification and characterization of subpopulations in undifferentiated ES cell culture. Development 135, 909-918 (2008).

26. Kim, $\mathrm{H}$. et al. Modulation of $\beta$-catenin function maintains mouse epiblast stem cell and human embryonic stem cell self-renewal. Nat Commun 4, 2403 (2013).

27. Pfaffeneder, T. et al. Tet oxidizes thymine to 5-hydroxymethyluracil in mouse embryonic stem cell DNA. Nat Chem Biol 10, 574-581 (2014).

28. Shirane, K. et al. Global Landscape and Regulatory Principles of DNA Methylation Reprogramming for Germ Cell Specification by Mouse Pluripotent Stem Cells. Developmental Cell 39, 87-103 (2016). 
29. Blaschke, K. et al. Vitamin $\llcorner C$ induces Tet-dependent DNA demethylation and a blastocyst-like state in ES cells. Nature 500, 222-226 (2013).

30. van Mierlo, G., Wester, R. A. \& Marks, H. A mass spectrometry survey of chromatin-associated proteins in pluripotency and early lineage commitment. Proteomics 19, e1900047 (2019).

31. Yagi, M. et al. Derivation of ground-state female ES cells maintaining gamete-derived DNA methylation. Nature 548, 224-227 (2017).

32. Choi, J. et al. Prolonged Mek1/2 suppression impairs the developmental potential of embryonic stem cells. Nature 548, 219-223 (2017).

33. Kalkan, T. et al. Tracking the embryonic stem cell transition from ground state pluripotency. Development 144, 1221-1234 (2017).

34. Wang, L. et al. Programming and Inheritance of Parental DNA Methylomes in Mammals. Cell 157, 979-991 (2014).

35. Lu, F., Liu, Y., Jiang, L., Yamaguchi, S. \& Zhang, Y. Role of Tet proteins in enhancer activity and telomere elongation. Genes Dev. 28, 2103-2119 (2014).

36. Hooper, M., Hardy, K., Handyside, A., Hunter, S. \& Monk, M. HPRT-deficient (Lesch-Nyhan) mouse embryos derived from germline colonization by cultured cells. Nature 326, 292-295 (1987).

37. Tsumura, A. et al. Maintenance of self-renewal ability of mouse embryonic stem cells in the absence of DNA methyltransferases Dnmt1, Dnmt3a and Dnmt3b. Genes Cells 11, 805-14 (2006).

38. Jekunen, A., Puukka, M. \& Vilpo, J. Exclusion of exogenous 5-methyl-2'-deoxycytidine from DNA in human leukemic cells: A study with [2-14C]- and [methyl-14C]5-methyl-2'-deoxycytidine. Biochemical Pharmacology 32, 1165-1168 (1983).

39. Maley, G. F., Lobo, A. P. \& Maley, F. Properties of an affinity-column-purified human deoxycytidylate deaminase. Biochimica et Biophysica Acta (BBA) - Protein Structure and Molecular Enzymology 1162, 161170 (1993).

40. de Saint Vincent, B. R., Déchamps, M. \& Buttin, G. The modulation of the thymidine triphosphate pool of Chinese hamster cells by dCMP deaminase and UDP reductase. Thymidine auxotrophy induced by CTP in dCMP deaminase-deficient lines. J. Biol. Chem. 255, 162-167 (1980).

41. Shen, J.-C., Rideout, W. M. \& Jones, P. A. The rate of hydrolytic deamination of 5-methylcytosine in doublestranded DNA. Nucl. Acids Res. 22, 972-976 (1994).

42. Ladstätter, S. \& Tachibana-Konwalski, K. A Surveillance Mechanism Ensures Repair of DNA Lesions during Zygotic Reprogramming. Cell 167, 1774-1787.e13 (2016).

43. Jiang, D., Wei, S., Chen, F., Zhang, Y. \& Li, J. TET3-mediated DNA oxidation promotes ATR-dependent DNA damage response. EMBO reports 18, 781-796 (2017).

44. Kweon, S.-M. et al. Erasure of Tet-Oxidized 5-Methylcytosine by a SRAP Nuclease. Cell Reports 21, 482494 (2017).

45. Robertson, A. B., Robertson, J., Fusser, M. \& Klungland, A. Endonuclease G preferentially cleaves 5hydroxymethylcytosine-modified DNA creating a substrate for recombination. Nucl. Acids Res. 42, 1328013293 (2014).

46. Liu, N. et al. Intrinsic and Extrinsic Connections of Tet3 Dioxygenase with CXXC Zinc Finger Modules. PLoS ONE 8, e62755 (2013).

47. Shipony, Z. et al. Dynamic and static maintenance of epigenetic memory in pluripotent and somatic cells. Nature 513, 115-119 (2014).

48. Ooi, S. K. et al. Dynamic instability of genomic methylation patterns in pluripotent stem cells. Epigenetics \& Chromatin 3, 17 (2010).

49. Chen, T., Ueda, Y., Dodge, J. E., Wang, Z. \& Li, E. Establishment and maintenance of genomic methylation patterns in mouse embryonic stem cells by Dnmt3a and Dnmt3b. Mol Cell Biol 23, 5594-605 (2003).

50. Bachman, M. et al. 5-Hydroxymethylcytosine is a predominantly stable DNA modification. Nat Chem 6, 10491055 (2014).

51. Bachman, M. et al. 5-Formylcytosine can be a stable DNA modification in mammals. Nat Chem Biol 11, 555557 (2015).

52. Rulands, S. et al. Genome-Scale Oscillations in DNA Methylation during Exit from Pluripotency. Cell Systems 7, 63-76.e12 (2018).

53. Tichy, E. D. et al. Mouse Embryonic Stem Cells, but Not Somatic Cells, Predominantly Use Homologous Recombination to Repair Double-Strand DNA Breaks. Stem Cells and Development 19, 1699-1711 (2010).

54. Tichy, E. D. et al. Mismatch and base excision repair proficiency in murine embryonic stem cells. DNA Repair 10, 445-451 (2011).

55. Maynard, S. et al. Human Embryonic Stem Cells Have Enhanced Repair of Multiple Forms of DNA Damage. STEM CELLS 26, 2266-2274 (2008). 
Spada et al.

\section{Figure Legends}

Figure 1. Drastic reduction of global genomic $\mathrm{mdC}$ oxidation and increase of $\mathrm{mdC}$ to mdU conversion upon transition to primed pluripotency.

a) Proposed de-modification pathways for genomic C5-cytosine modifications and expected labelled $\mathrm{mdC}$ derivatives upon metabolic labelling with $m+4$ Met. b) Nucleoside isotopologues used as internal standards for UHPLC-MS ${ }^{2}$ analysis. Black balls in the ribose represent ${ }^{13} \mathrm{C}$ atoms. c and $\mathrm{d}$ ) Time course analysis of genomic $\mathrm{dC}$ derivatives upon metabolic labelling with $\mathrm{m}+4 \mathrm{Met}$ under naïve (c; a2i/LIF) and priming (d; serum/CR) conditions. The labelling/time course schedules are shown at the top. Global levels of unlabelled (pale yellow) $m d C$ and $m+4 d C$ (dark yellow), $h m+3 C$ (blue) and $f+2 C$ (green) are shown as mean and standard deviation of three technical replicates. See also Fig. S3 for an independent biological replicate. LOD = limt of detection.

Figure 2. Turnover of genomic $\mathrm{mdC}$ and its derivatives under naïve and priming conditions

a) Workflow of $m+4 M e t$ and $d T+12$ pulse-chase experiments with wild type mPSCs under naïve and priming conditions. b) Global profiles of the indicated labelled cytosine derivatives and $\mathrm{dT}+12$ in the genome of naïve (a2i/LIF; left) and primed mPSCs (serum/CR; right and inset) upon chase of $m+4$ Met. Upper panels show values relative to the highest level for each modification. Dashed lines represent first order decay fitted curves. Lower panels show absolute values of genomic m+4Met (note the different scales for $m+4 d U$ in the two conditions). Mean values and standard deviation of triplicate measurements are shown. c) Percent of difference between fold changes of $\mathrm{dT}+12$ and $\mathrm{m}+4 \mathrm{dC}$ in wt mPSCs under priming conditions. Shown are mean and standard deviation of three independent biological replicates.

Figure 3. The active component of $\mathrm{mdC}$ turnover in primed pluripotent cells largely depends on Tet proteins.

$\mathrm{m}+4$ Met and $\mathrm{dT}+12$ pulse-chase experiment with parental and Tet TKO E14tg2a mPSCs under priming conditions (serum/CR). Profiles of global genomic levels of the indicated labelled cytosine derivatives and $\mathrm{dT}+12$ are shown. Upper and mid panels show $\mathrm{m}+4 \mathrm{dC}$ and $\mathrm{dT}+12$ values relative to the highest data point for each modification. Upper panels display magnifications of mid panels fields in dotted frames. Dashed lines represent first order decay fitted curves. Lower panels display global genomic levels of $m+4 d U$. 
Figure 4. All detectable mdC deamination takes place in the soluble pool after DNA repair.

a) Potential pathways for the conversion of genomic $m+4 d C$ into genomic $m+4 d U$. b) wt mPSCs were primed under CHIR/IWR1 (serum/CR) conditions in the presence of $m+4 \mathrm{Met}$ and growing concentrations of unlabelled dT in the culture medium as indicated. c) wt and CD-DKO clones mPSCs were grown under serum/LIF conditions in the presence of $\mathrm{m}+4$ Met. d) wt and CD-DKO clone \#40 mPSCs were metabolically labelled with $\mathrm{m}+4 \mathrm{Met}$ under serum/CR priming conditions. In b-d priming and labelling was for five days. Global genomic contents of $\mathrm{mdC}, \mathrm{m}+4 \mathrm{dC}$ (upper panels) and $\mathrm{m}+4 \mathrm{dU}$ (lower panels) are shown as mean and standard deviation of three technical replicates. LOD = limit of detection.

Figure 5. mdC turnover through oxidation-independent DNA repair is developmentally regulated.

a) Cultures of mPSCs in naïve (a2iL = a2i/LIF) and primed states (CR = CHIR/IWR1; FA = FGF-2/Activin A; CDM = Chemically Defined Medium), primary (pmEFs) and immortalized mouse embryonic fibroblasts (imEFs and NIH3T3), HeLa and mPSC-derived neural stem cells (mNSCs) were labelled for the indicated number of days (d) with $m+4 M e t$. Asterisks indicate cases where distinct parallel cultures in the absence and presence of $m+4 M e t$ were used to measure global levels of genomic $\mathrm{mdC}$ and $\mathrm{m}+4 \mathrm{dU}$, respectively. In all other cases $m d C, m+4 d C$ and $m+4 d U$ were measure in the same culture labelled with $m+4$ Met. Mean and standard deviation of three technical replicates are shown. b) Schematic summary of the $\mathrm{dC}$ modification turnover pathways identified in primed pluripotent cells. Deamination of genomic mdC followed by BER could not be detected and may occur only at levels below the sensitivity of our assay. 
Spada et al.

\section{Methods}

\section{Cell culture}

Medium components were from Sigma unless specified otherwise. Basal medium for mPSC culture was DMEM high glucose containing 10\% FBS (Pan-ES, Pan-Biotech), 2 mM LAlanyl-L-Glutamine, 1x MEM Non-essential Amino Acid Solution and $0.1 \mathrm{mM} \beta$ mercaptoethanol. mPSC lines were routinely maintained in basal medium supplemented with $1000 \mathrm{U} / \mathrm{mL}$ LIF (ORF Genetics), $3 \mu \mathrm{M}$ CHIR99021 and $7.5 \mu \mathrm{M}$ CGP77675 (a2i). For adaptation to 2i/LIF conditions CGP was replaced with $1 \mu \mathrm{M}$ PD0325901. For priming, mPSC basal medium was supplemented with $1.5 \mu \mathrm{M}$ CHIR99021 and IWR1-endo at $2.5 \mu \mathrm{M}$ as previously reported ${ }^{26,56}$. For experiments under serum/LIF conditions basal medium was supplemented exclusively with $1000 \mathrm{U} / \mathrm{ml}$ LIF. Small molecule inhibitors were purchased from Selleckchem (CHIR, PD, IWR1), Axon Medchem (CHIR, CGP, PD, IWR1) or MedChemExpress (CHIR). Gelatin coating was used for all mPSCs cultures in serum containing medum, except for PR8 cultures where coating with $2.2 \mu \mathrm{g} / \mathrm{cm}^{2}$ Laminin 521 (BioLamina) was necessary. For metabolic labelling experiments with $m+4 M e t$ under all conditions mPSC basal medium was generated with $0.2 \mathrm{mM} \mathrm{L-Methionine-} \mathrm{and} \mathrm{L-Cysteine-}$ free DMEM (Sigma or Life Technologies) supplemented with $\mathrm{m}+4 \mathrm{Met}\left(\left[\right.\right.$ methyl- $\left.{ }^{13} \mathrm{C}, \mathrm{d}_{3}\right]$ ) and L-Cysteine. EpiSCs in chemically defined serum-free medium (CDM) were derived from the Oct4-YFP reporter mPSC line OLY2-1 ${ }^{25}$. These were first adapted to CDM supplemented with CHIR, PD and LIF as reported above, then transferred to CDM supplemented with CHIR, CGP, $12 \mathrm{ng} / \mathrm{ml}$ FGF-2 (Miltenyi Biotec) and $20 \mathrm{ng} / \mathrm{ml}$ Activin A (PeproTec) and maintained in the latter medium by passaging as small cell clusters using 5 mM EDTA in Hank's balanced salt solution without $\mathrm{Mg}^{2+}, \mathrm{Ca}^{2+}$ and sodium carbonate supplemented with $10 \mathrm{mM}$ HEPES buffer. CDM was DMEM high glucose supplemented with 1x NEAA, $2 \mathrm{mM} \mathrm{L-}$ Alanyl-L-Glutamine, $15 \mu \mathrm{g} / \mathrm{ml}$ human recombinant Insulin, $10 \mu \mathrm{g} / \mathrm{ml}$ human holo-Transferrin (Merck), $12.5 \mathrm{mg} / \mathrm{ml}$ AlbuMAX I (Life Technologies) $0.7 \mu \mathrm{g} / \mathrm{ml}$ vitamin B12, $1.8 \mathrm{ng} / \mathrm{ml}$ biotin, $0.75 \mu \mathrm{M} \mathrm{ZnSO}_{4}$ and $2.6 \mathrm{nM} \mathrm{CuSO}_{4}$. Laminin 521 coating was used for all mPSC cultures in CDM. The neural stem cell line ENC1 was generated and cultured as previously described ${ }^{46}$. ENC1 cells were labelled with $m+4$ Met $\left(\left[\right.\right.$ methyl $\left.\left.{ }^{13} \mathrm{C}, \mathrm{d}_{3}\right]\right)$ in DMEM/F-12 generated with L-Methionine-free DMEM supplemented with $\mathrm{N}^{57}$ and $20 \mathrm{ng} / \mathrm{ml}$ each of FGF-2 and EGF (PerpoTech). Primary mEFs (CF-1, Applied StemCell) were cultured in mPSC basal medium. Somatic cell lines were cultured in DMEM containing 10\% FBS (Life Technologies), $2 \mathrm{mM}$ L-glutamine and $0.1 \mathrm{mM} \beta$-mercaptoethanol. 
Spada et al.

\section{Generation of CD-DKO mPSCs by CRISPR/Cas9 editing}

Single guide RNAs were designed over exon1 and 4 of Cda and Dctd, respectively (Fig. S5), using CRISPR Design Tool (http://crispr.mit.edu/). Oligonucleotides used for construction of Cas9/gRNA expression vectors are listed in Supplementary Table 1. The oligonucleotides were annealed and cloned in the Bbsl site of pSpCas9-2A-Puro (PX 459; Addgene Plasmid 48139 ${ }^{58}$. Cas9/gRNA expression vectors targeting both Cda and Dctd were co-transfected in wt J1 mPSCs using Lipofectamine 2000 (Life Technology) according to the manufacturer's instructions. Two days after transfection cultures were selected with $1 \mu \mathrm{g} / \mathrm{ml}$ puromycin for two days and subcloned by limiting dilution. Clones were screened using the Surveyor Mutation Detection Kit (Transgenomics) and potential compound biallelically targeted clones were subject to sequencing. For reverse transcription-PCR, RNA was isolated with the ZRDuet DNA/RNA MiniPrep Kit (Zymo Research) and first strand cDNA synthesis was as previously described ${ }^{59}$. Primers were used to amplify the Cda cDNA are reported in Supplementary Table 1. For western blot analysis of Dctd whole cell extracts and purified human recombinant DCTD protein ${ }^{60}$ were probed with a rabbit anti-DCTD antibody ${ }^{39}$.

\section{Isolation of genomic DNA and UHPLC-MS ${ }^{2}$ analysis}

Isolation, digestion and UHPLC-MS ${ }^{2}$ analysis of gDNA samples were performed as described in Traube et al. ${ }^{61}$ with the following modifications: for every technical replicate $4 \mu \mathrm{g}$ of gDNA were digested and analysed; the enzyme mixture including S1 Nuclease, Antarctic Phosphatase and snake venom Phosphodiesterase was used for digestion; the digestion mixture was supplemented with $0.8 \mu \mathrm{M}$ Tetrahydrouridine (Abcam) to inhibit potential cytidine deaminase activity in enzyme preparations.

\section{Chemical synthesis}

For a description of the synthesis of labelled nucleosides see Supplementary Methods in Supplementary Information file.

\section{Methods-specific references}

56. Zhou, X., Chadarevian, J. P., Ruiz, B. \& Ying, Q.-L. Cytoplasmic and Nuclear TAZ Exert Distinct Functions in Regulating Primed Pluripotency. Stem Cell Reports 9, 732-741 (2017).

57. Nichols, J. \& Ying, Q.-L. Derivation and propagation of embryonic stem cells in serum- and feeder-free culture. in Embryonic Stem Cell Protocols (ed. Turksen, K.) vol. 329 91-98 (Humana Press, 2006).

58. Ran, F. A. et al. Genome engineering using the CRISPR-Cas9 system. Nat. Protocols 8, 2281-2308 (2013).

59. Rahimoff, R. et al. 5-Formyl- and 5-Carboxydeoxycytidines Do Not Cause Accumulation of Harmful Repair Intermediates in Stem Cells. J. Am. Chem. Soc. 139, 10359-10364 (2017).

60. Weiner, K. X., Weiner, R. S., Maley, F. \& Maley, G. F. Primary structure of human deoxycytidylate deaminase and overexpression of its functional protein in Escherichia coli. J. Biol. Chem. 268, 12983-12989 (1993).

61. Traube, F. R. et al. Isotope-dilution mass spectrometry for exact quantification of noncanonical DNA nucleosides. Nature Protocols 14, 283 (2019). 
2 Metabolic labelling with $\left[{ }^{13} \mathrm{C}, \mathrm{D}_{3}\right.$-methyl]-methionine alias [m+4]-Met<smiles>[2H]C([2H])([2H])[13CH2]CC[C@H](N)C(=O)O</smiles>

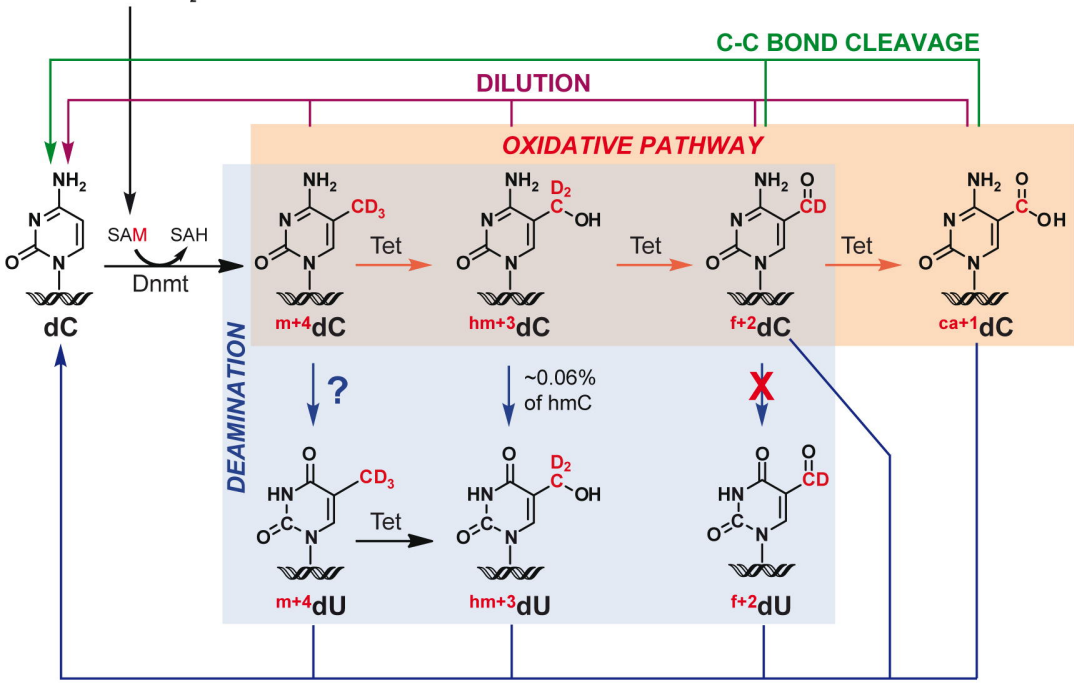

BASE EXCISION REPAIR

b

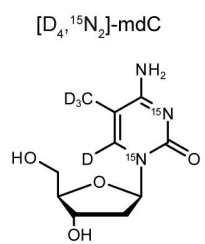

$\left[{ }^{13} \mathrm{C}_{5},{ }^{15} \mathrm{~N}_{2}\right]-\mathrm{hmdC}$
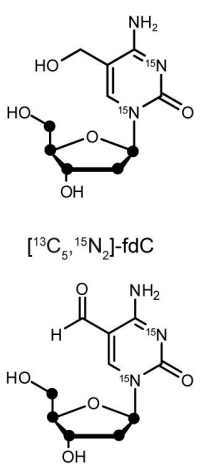

$\left[{ }^{3} \mathrm{C}_{5},{ }^{15} \mathrm{~N}_{2}\right]-d \mathrm{~T}$

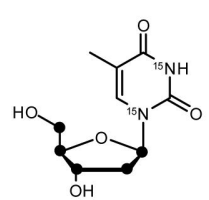

C
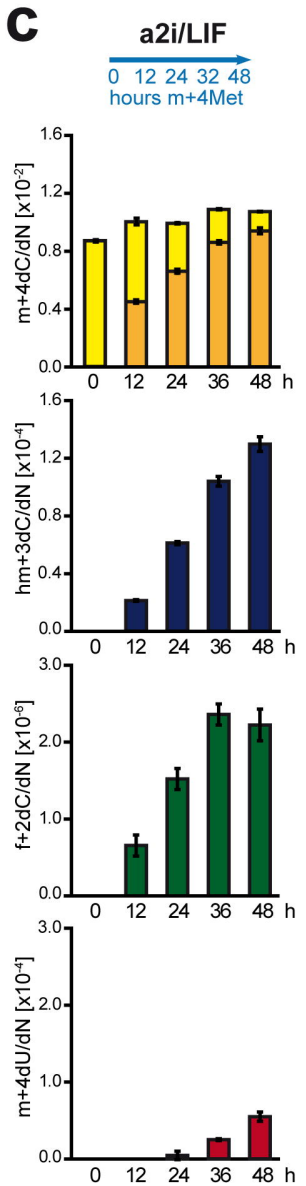
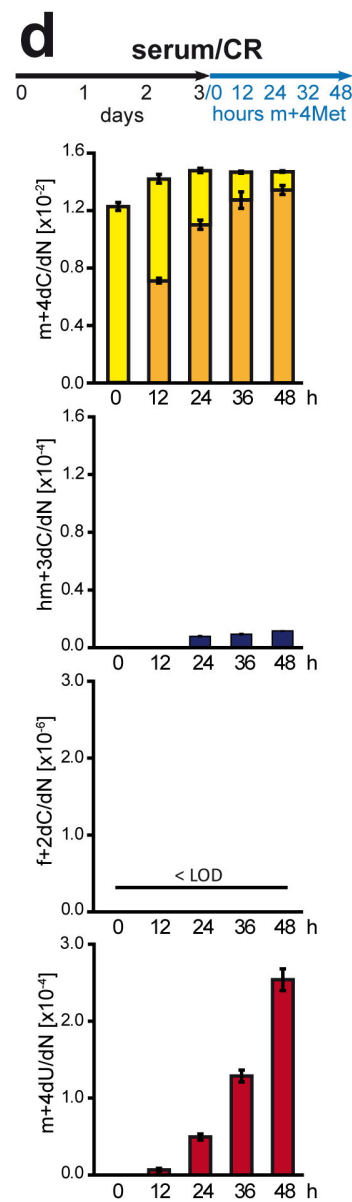


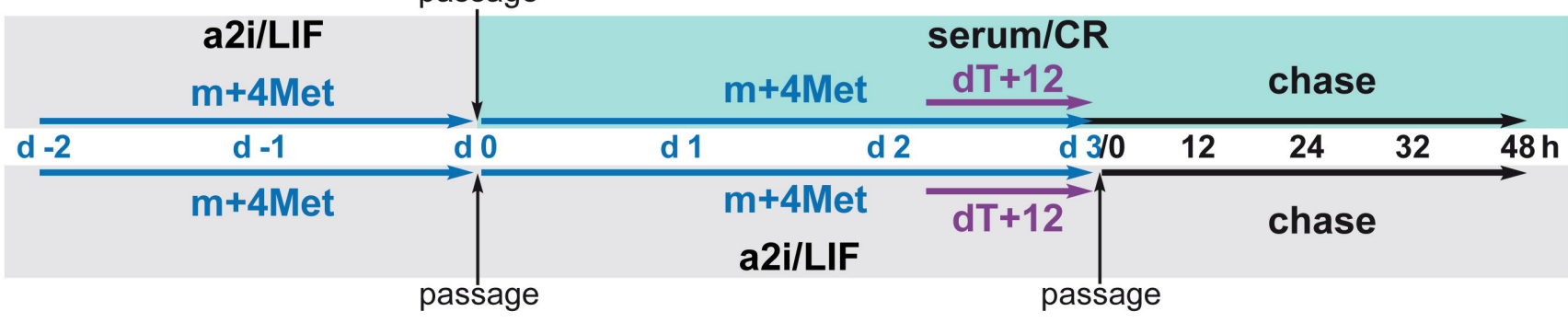

b

\section{a2i/LIF}
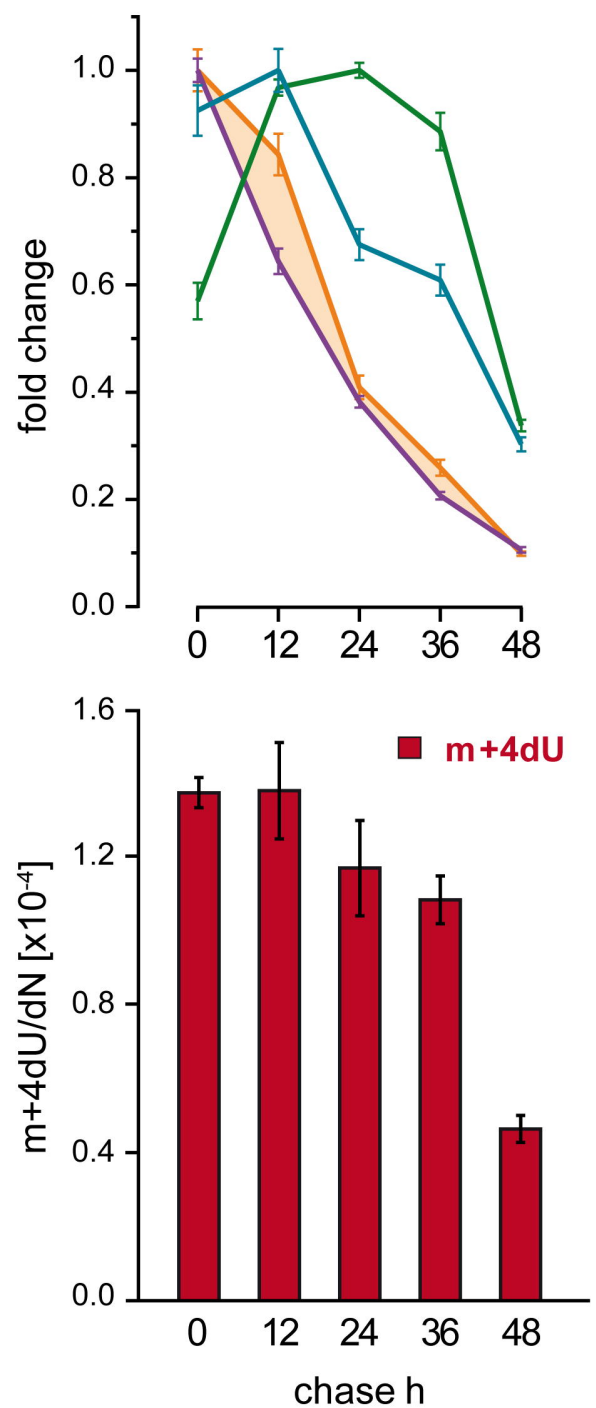

serum/CR
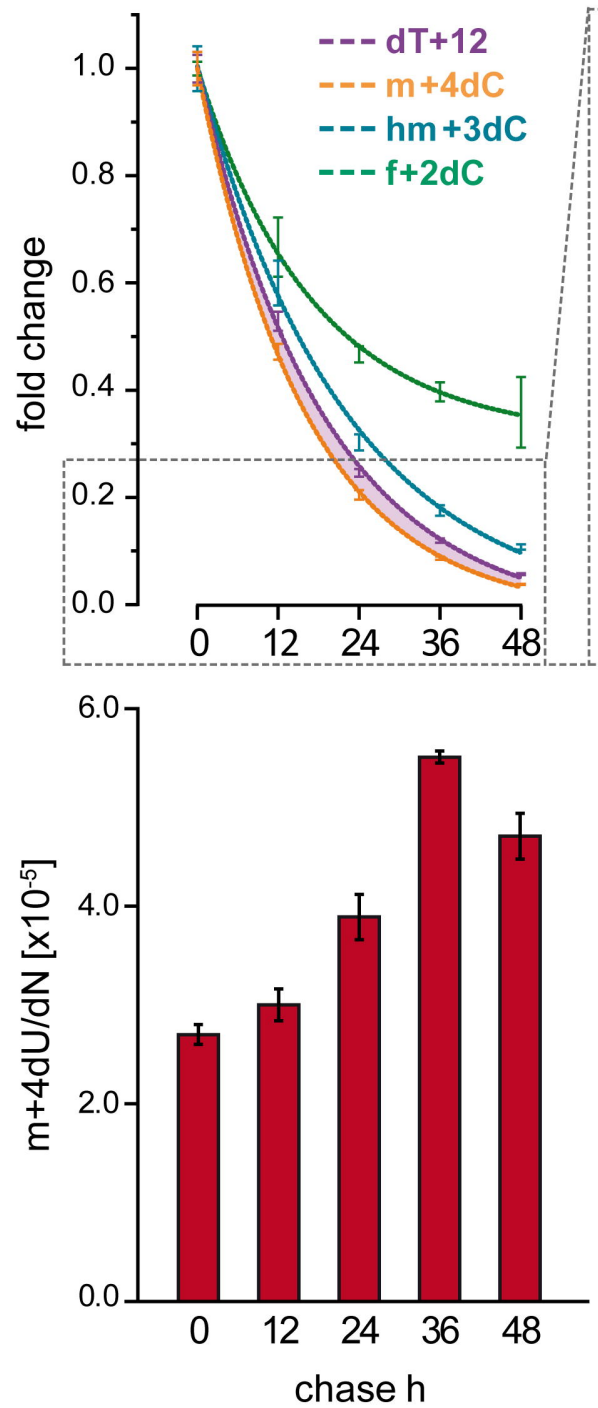

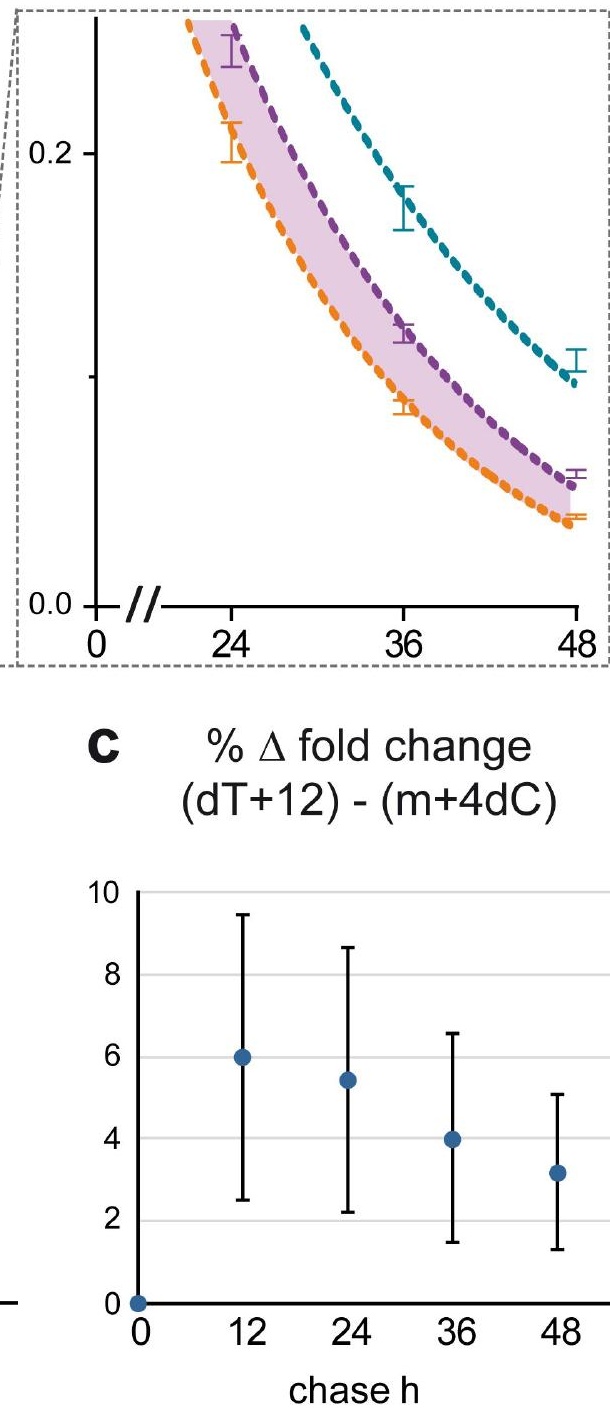


E14tg2a

Tet TKO E14tg2a

\section{$m m+4 d C \ldots d T+12 \square m+4 d U$}

0
00
$\frac{1}{0}$
$\frac{1}{0}$
$\frac{0}{0}$
$\frac{0}{4}$

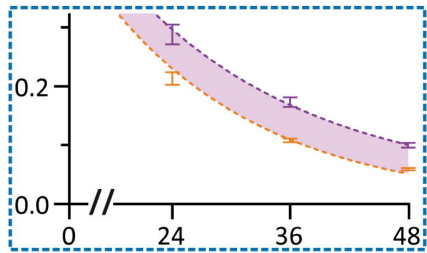

0
0
0
0
0
4
0
0
4
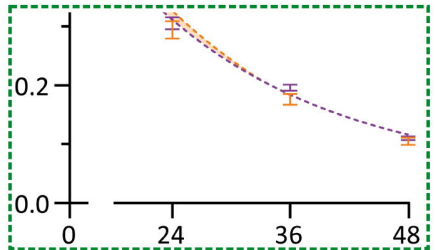

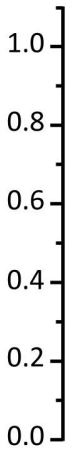

36

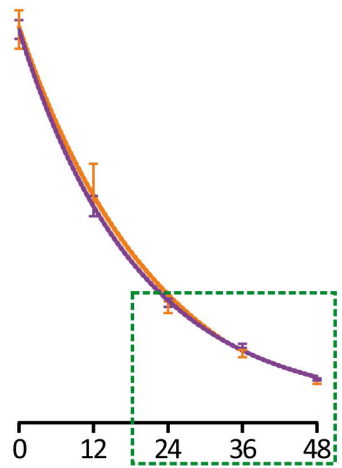

2.8

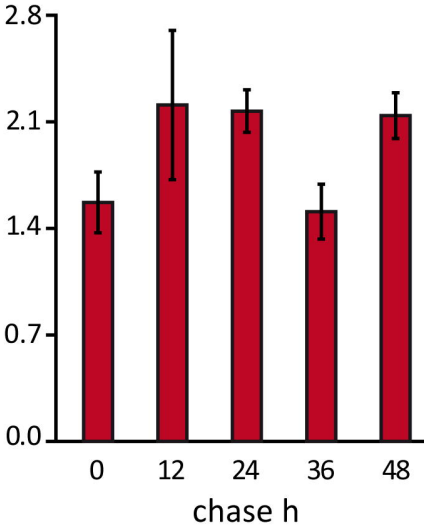



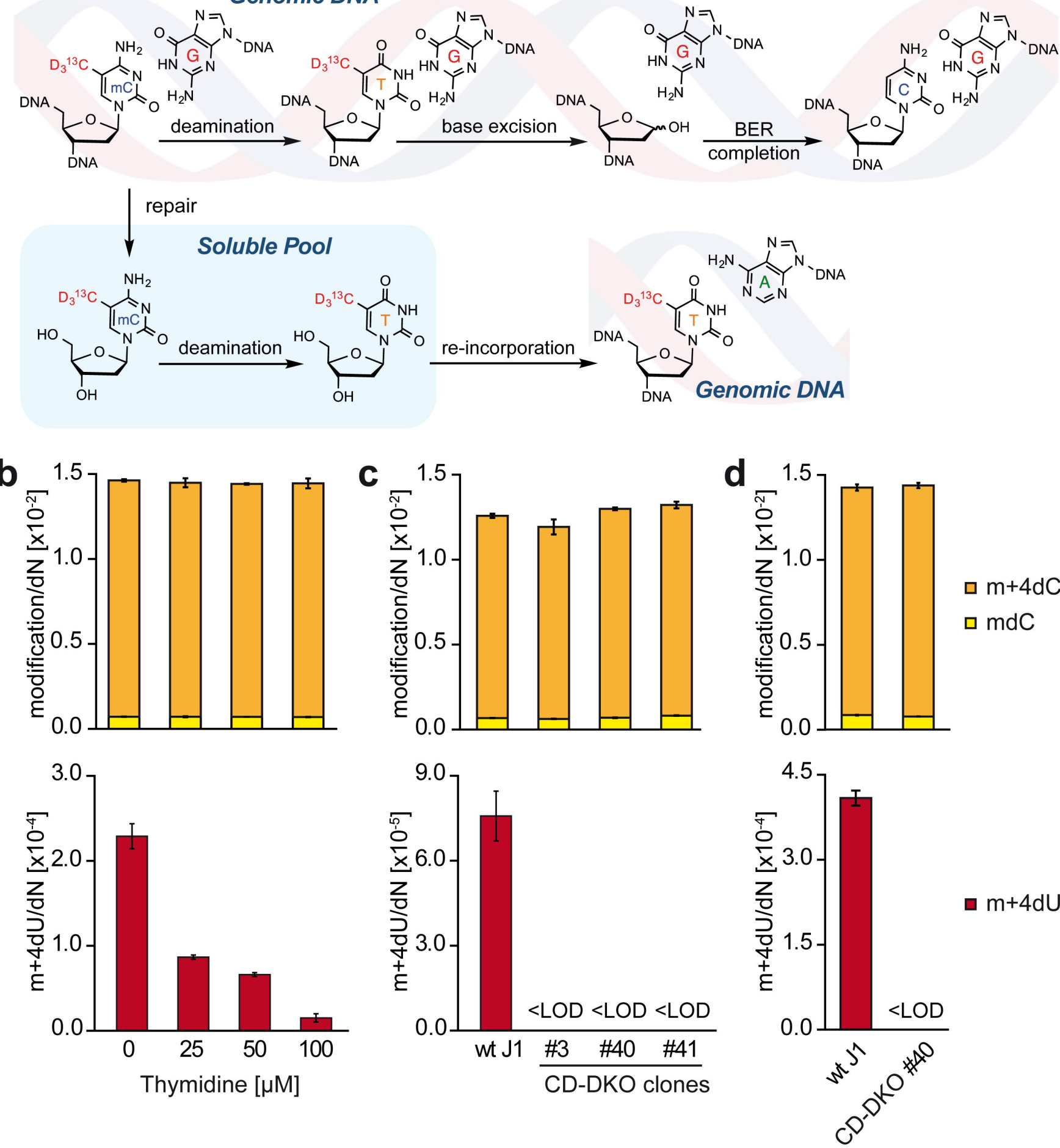

$\square \mathrm{m}+4 \mathrm{dC}$
$\square \mathrm{mdC}$

a $\mathrm{m}+4 \mathrm{dU}$ 

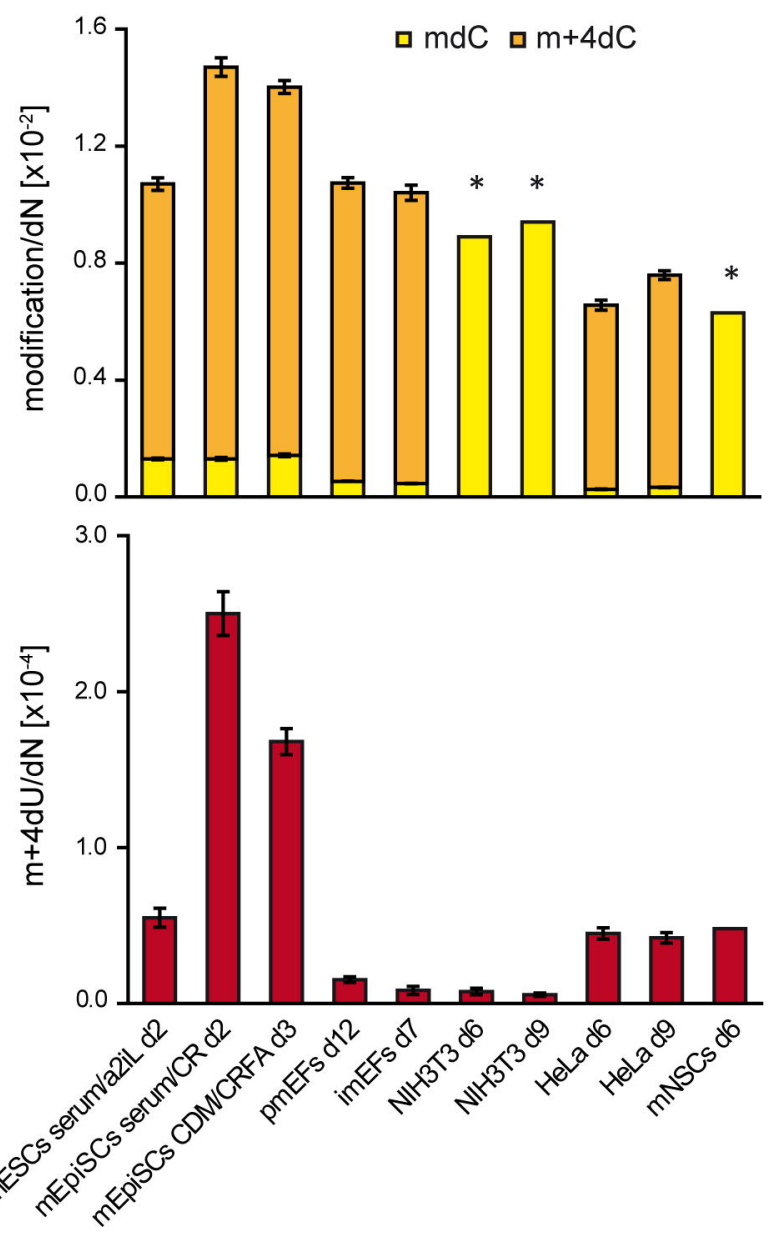

b
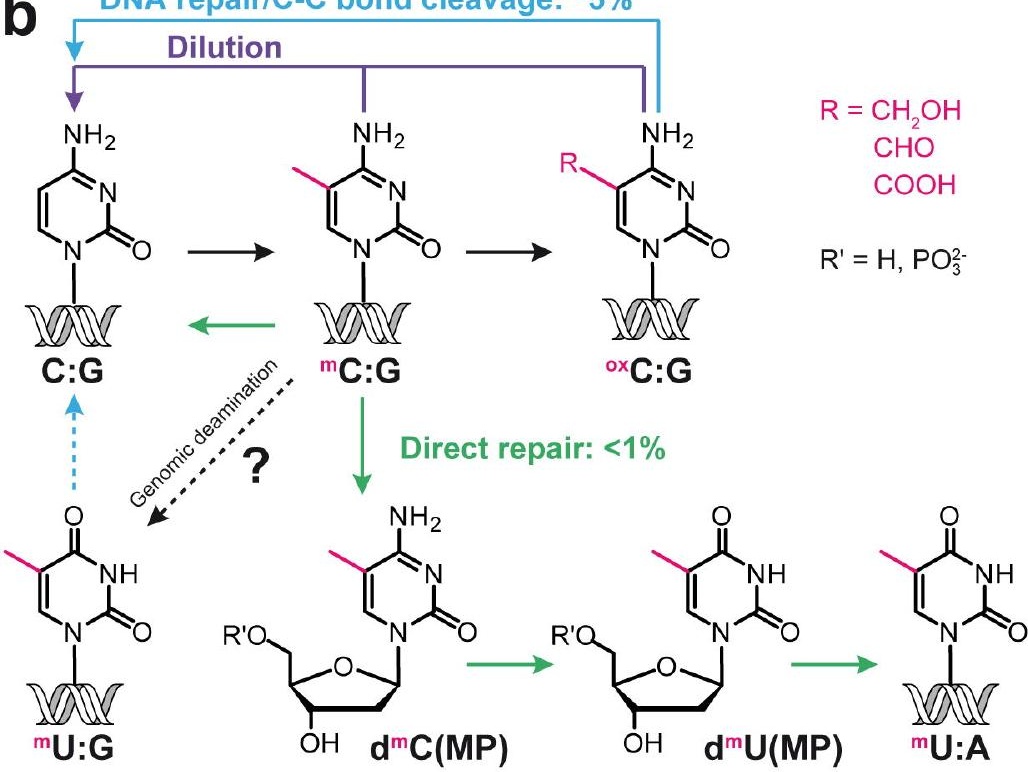